\title{
Correction to: 5G Security: Concepts and Challenges
}

Poorna Pravallika Sriram, Hwang-Cheng Wang, Hema Ganesh Jami, and Kathiravan Srinivasan

\section{Correction to:}

Chapter 1 in: D. N. K. Jayakody et al. (eds.),

5 G Enabled Secure Wireless Networks, https://doi.org/10.1007/978-3-030-03508-2_1

The original version of this chapter was inadvertently published with the incorrect affiliation of "Hema Ganesh Jami". The affiliation detail has now been corrected from "National Ilan University, Yilan City, Taiwan" to "Vel Tech Rangarajan Dr. Sagunthala R\&D Institute of Science and Technology, Chennai, India". 